\title{
CONTRIBUCIÓN DE LA FOTOVOZ A LA INVESTIGACIÓN DE ESTUDIO DE CASOS EN EDUCACIÓN
}

\author{
María Jesús Romera Iruela
}

Universidad Complutense, Madrid - mjromera@ucm.es

\begin{abstract}
Resumen. El artículo se sitúa en el nivel meta-teórico de la Epistemología de la investigación pedagógica, en su apertura a una pluralidad metódica que posibilite la justificación del conocimiento que realmente eduque. Analiza, desde ese contexto, una vinculación de técnicas que, procedentes de los campos de la Antropología y la Sociología, han originado la fotovoz y realiza una indagación acerca de su incorporación a la investigación educativa de estudio de casos. El procedimiento seguido está constituido por la reflexión epistemológica, la metodología documental y el análisis de contenido. Los resultados aportan claridad a la confusión existente en torno a la fotovoz, así como sus potencialidades y límites, radicando en su epistemología el más esencial de los últimos. Además, ponen de manifiesto la escasa incorporación de este procedimiento en los estudios de casos en el ámbito de la educación, dando a conocer las temáticas específicas, sus usos en esta forma de investigación y sus principales contribuciones. Se concluye con una propuesta de ampliar este enfoque de la foto-elicitación mediante el desarrollo de otro inscrito en el paradigma de la investigación participativa.
\end{abstract}

Palabras clave: Epistemología; Metodología; Investigación Educativa; Estudio de Casos; Fotovoz.

\section{CONTRIBUTION OF THE PHOTOVOICE TO CASE STUDY RESEARCH IN EDUCATION}

\begin{abstract}
The article is framed at the meta-theoretical level of the Epistemology of Pedagogical Research, in its openness to a methodical plurality that makes possible the justification of the knowledge that really educates. It analyzes, from this context, a linkage of techniques that, coming from the fields of Atropology and Sociology, have originated the photovoice and it makes an inquiry about its incorporation to the educational research of case study. The procedure followed consists of epistemological reflection, documentary methodology and content analysis. The results bring clarity to the existing confusion about the photovoice, as well as its potentialities and limits, the most essential of the latter lies in its epistemology. They also show the low incorporation of this procedure in the case studies in the field of education, making known the specific themes, the uses of this form of research and their main contributions. It concludes with a proposal to extend this photo-elicitation approach through the development of another one framed in the paradigm of participatory research.
\end{abstract}

Keywords: Epistemology; Methodology; Educational Research; Case Study; Photovoice.

\section{INTRODUCCIÓN - PLANTEAMIENTO DEL OBJETO DE ESTUDIO}

La metodología de la investigación pedagógica, en su pretensión de llegar a la justificación del conocimiento que realmente educa a los seres humanos, se ha ido habiendo a una búsqueda de métodos y técnicas que hacen posible el logro progresivo de la mencionada finalidad, buscando, al mismo tiempo, su propia justificación mediante el análisis reflexivo y crítico de su racionalidad con sus principios epistemológicos, que son los que los fundamentan y orientan. De ahí que en la indagación de la acción educativa que, necesariamente incluye cualidad y cantidad, no puedan dejar de estar presentes los 
procedimientos cualitativos y cuantitativos, porque los requiere la índole de su objeto de estudio, cuyo conocimiento ha de integrar lo idiográfico y lo nomotético.

El estudio de casos es uno de los enfoques presentes en la investigación educativa y el caso único como universo de investigación ha sido visto como un nuevo paradigma de la del campo pedagógico (Vázquez, 1981). Si bien no se ha llegado a una definición y comprensión única de este enfoque de casos y los debates están presentes en las trayectorias de su desarrollo, como Schwandt y Gates (2018) han puesto de relieve, nos parece significativa la síntesis expresada por dichos autores de que vista, colectivamente, "toda la investigación de estudio de casos existe para abordar la dialéctica que se encuentra en el corazón de la comprensión -una investigación en curso de lo empírico para refinar lo teórico y de lo teórico para comprender y explicar mejor lo empírico" (p. 354). Sin duda, todo un quehacer de enorme valor.

Los principales usos de los estudios de casos, que siendo diferentes no se excluyen mutuamente, se han agrupado en cuatro categorías: "(1) descripción, (2) generación de hipótesis o desarrollo de teorías, (3) prueba de hipótesis y teorías y (4) desarrollo de la teoría normativa" (Schwandt \& Gates 2018, p. 346). Ellos están en consonancia con una comprensión profunda de las entidades y de los fenómenos que son el objeto de estudio en las investigaciones.

Las técnicas presentes y posibilitadoras de los estudios de casos son múltiples y variadas, situándose en lo cualitativo y lo cuantitativo. La investigación visual, como indican Margolis y Zunjardwad (2018) atraviesa eficazmente las brechas entre la referida división convencional y amplía las posibilidades de implementación en una variedad de campos.

En los últimos años ha surgido la fotovoz y ha sido sistematizada por Lapenta (2011) como uno de los enfoques de la foto-elicitación. Estos facilitan y posibilitan el acceso a las propias experiencias de los seres humanos en sus distintos contextos, conectando con sus recuerdos, sentimientos e ideas y, por ello, son una vía de acceso al significado y sentido de las acciones humanas y sociales. Sin embargo, en torno a ella, se ha advertido confusión terminológica, así como falta de precisión metodológica y conceptual, junto con algunas limitaciones.

La cuestión origen y foco de atención de este trabajo es estudiar en profundidad la fotovoz, desde la racionalidad del paradigma de investigación en el que se inscribe, e indagar en su 
incorporación en la investigación de estudio de casos en educación. Nuestro objetivo dual es realizar, en primer lugar, el estudio meta-teórico de la fotovoz, analizando sus potencialidades y sus límites, sus presupuestos y sus consecuencias, así como su valor para el incremento del conocimiento, sugiriendo la posibilidad de optimización desde el paradigma de la investigación participativa, y, en un segundo y último lugar, la indagación acerca de su incorporación en la investigación educativa de estudio de casos, poniendo de manifiesto su aportación.

\section{METODOLOGÍA}

La conceptualización de la fotovoz que aportamos se basa en el examen de los trabajos en los que se ha dado a conocer y en la revisión de otros posteriores que la han considerado, efectuando, al hilo de la misma, nuestra reflexión meta-teórica centrada en el paradigma en el que se fundamenta y guía su aplicación, aportando precisión conceptual y metodológica.

La indagación sobre la aplicación de la fotovoz a la investigación educativa de estudio de casos tiene como punto de partida la realización de diversas búsquedas documentales en las principales bases de datos multidisciplinarias y especializadas: Web of Science, Scopus, TESEO, Dialnet, ERIC y REDINED, a las que se añadió un sondeo en Google Académico. La interrogación de estas bases se hizo, teniendo presente la estructura de la mayoría de ellas y la terminología propia de la necesidad de información, mediante la elaboración de un perfil de búsqueda en lenguaje natural, constituido por la intersección, con el uso del operador booleano "y", de estas palabras clave: fotovoz (Photovoice), estudio de casos (case study) y educación (education), que se incorporó a la modalidad de búsqueda avanzada para la recuperación de la información. Una vez obtenidas las referencias de los documentos y su depuración, como inadecuaciones o repeticiones de una misma investigación en varios documentos, se procedió a la recopilación de los textos de los trabajos cuando no podían obtenerse desde esas bases de datos.

Finalmente, aplicamos el análisis de contenido a los aspectos considerados en el objeto de estudio de este trabajo: evolución de los trabajos que han incorporado la fotovoz en su investigación de estudio de casos, canales formales e informales en los que se ha difundido esta investigación, naturaleza del objetivo al que se orienta la fotovoz en esos estudios de casos, unidad en los casos con las temáticas de la realidad estudiada, uso de dichos estudios con sus principales contribuciones. En la codificación y el establecimiento de las categorías se procedió siguiendo la vía deductiva, salvo en la identificación de los tipos de 
casos y los temas estudiados, que se obtuvieron por una vía inductiva, fundamentada en los datos referentes a los objetos de estudio de las investigaciones, por no existir tipologías en los trabajos previos, si bien tuvimos presente la revisión por Doval (2015). El análisis cronológico cubrió la totalidad de los años, que se agruparon en intervalos quinquenales. La codificación de los canales de difusión de las investigaciones se realizó en función de una clasificación del proceso de comunicación científica presentada por Cordón et al. (2016), que consta de dos categorías con varias formas. Estas, y los principales canales, son las siguientes: Publicación científica (comunicaciones a congresos, foros, blogs, correos, memorias de investigación, tesis doctorales y obras en Internet) y Edición científica (monografías científicas, actas de congresos, revistas científicas, tesis doctorales y bases de datos). La identificación de los objetivos de la fotovoz en los estudios de casos se estableció de acuerdo con la clasificación tradicional de los procedimientos científicos, que cuenta con dos vertientes o categorías: heurística y didáctica. En el examen de los usos de los estudios de casos se aplicó la reciente tipología de Schwandt y Gates (2018), que los sistematiza en las cuatro categorías que ya fueron citadas con anterioridad. La asignación a las categorías en todos los análisis fue mutuamente excluyente, excepto en las de esta última tipología, por no tener, necesariamente, dicho carácter. La interpretación de los datos se elaboró desde el marco teórico del presente trabajo.

\section{CONCEPTUALIZACIÓN Y ANÁLISIS METODOLÓGICO DE LA FOTOVOZ}

De conformidad con nuestra investigación teórico-metodológica, nos vamos a centrar en el origen de la fotovoz, en su delimitación conceptual, clarificando algunas imprecisiones que se mencionan en la bibliografía, en su procedimiento, en sus potencialidades y limitaciones, así como en su incidencia en la educación. Nuestro análisis desde la perspectiva paradigmática se hará al hilo de dicha elaboración meta-teórica.

\subsection{Origen y conceptualización de la fotovoz}

El reconocimiento del origen de la fotovoz en los trabajos en colaboración de Wang es unánime en la literatura especializada en el tema. En un artículo, publicado en 1996, esta autora, junto con Y. Ling y L. Ling, comunican un estudio de caso, centrado en mujeres rurales chinas implicadas en un proyecto de promoción de la salud comunitaria, que utiliza fotovoz para evaluar la adecuación del mismo. Este documento contiene ya una definición 
que, un año después, va a ser precisada en un nuevo trabajo, ahora realizado con Burris, dedicado a esta metodología, siendo conceptuada en estos términos:

Proceso por el que las personas pueden identificar, representar y mejorar su comunidad a través de una técnica fotográfica específica. Confía las cámaras a manos de las personas para posibilitarlas actuar como registradoras y potenciales catalizadoras del cambio en sus propias comunidades. Utiliza la inmediatez de la imagen visual para aportar evidencia y promover un medio participativo efectivo para compartir experiencia y conocimiento. (p. 369)

La caracterización del proceso que ofrecen estas autoras es rica en matices, poniendo de manifiesto su gran flexibilidad junto con la posibilidad de su adaptación, más allá de los principales objetivos que aportan, a otros propósitos participativos, a diversos grupos y comunidades y a diferentes asuntos de salud pública. Al leer este trabajo comprobamos la utilización indistinta del término "fotovoz" como método, método participativo, técnica y herramienta, lo cual pensamos que ya produce la confusión referida y contenida en las publicaciones posteriores. A ella nos referiremos más adelante, indicando, ahora, la puntualización de las autoras al manifestar que, en la fotovoz, no se trata sólo de utilizar las fotografías para contar o enseñar algo, sino que ellas han de posibilitar conocer las perspectivas de los participantes que las realizan acerca de un problema o asunto comunitario, o sea, su experiencia o saber individual y colectivo a través del diálogo, con el propósito de llegar a los líderes sociales y a los responsables políticos para producir cambio hacia una mejora de la comunidad.

Como práctica basada en la producción de conocimiento, los principales objetivos que sus creadoras formularon son:

1. Posibilitar a las personas registrar y reflejar las fortalezas y los asuntos de su comunidad.

2. Promover diálogo crítico y conocimiento acerca de las cuestiones importantes a través de la discusión de las fotografías en grupos pequeños y grandes.

3. Ilegar hasta los responsables políticos (Wang \& Burris, 1997, p.370)

El marco conceptual desde el que se desarrolla la fotovoz está formado por las siguientes fuentes:

1. La literatura teórica sobre educación para la conciencia crítica, destacando la pedagogía de Paulo Freire.

2. La teoría feminista.

3. Un enfoque de fotografía documental basado en la comunidad. 
4. Los principios de promoción de la salud. (Wang \& Burris, 1979, p. 369; Wang, 2006, p. 148).

Teniendo presente que, temáticamente, la fotovoz se ha creado en el ámbito de la educación para la salud y que la fotografía es el punto de partida en ella, vamos a considerar las dos primeras fuentes para, con ellas, argumentar su inscripción en el paradigma crítico.

Wang y Burris $(1994,1997)$ han destacado las ideas de problematización, creación de una conciencia crítica, empoderamiento y transformación social de Paulo Freire. En la metodología de alfabetización ideada por este pedagogo crítico se utilizaban los dibujos lineales y las fotografías. En la misma línea de la educación para la liberación de los oprimidos, la fotovoz da la cámara, a mujeres rurales, niños, trabajadores de base y otros miembros con poco acceso a quienes deciden sobre sus vidas, para que documenten lo que sucede en una comunidad o población. Desde las fotografías y el diálogo crítico se posibilita el desarrollo del conocimiento y la transformación social. Así, la dialéctica entre la teoría y la práctica de este enfoque orienta a la fotovoz.

Con respecto a la segunda fuente, Wang y Burris (1997) valoran que la teoría y la práctica feministas han iluminado el sesgo masculino que ha influido en la investigación participativa. Esta corriente, con su desarrollo, también se inscribe en el paradigma crítico. En nuestros días, el supuesto distintivo de este marco meta-teórico crítico es el de la naturaleza de la investigación determinada por el valor, en el que se sitúan distintas corrientes de pensamiento.

Desde nuestra consideración meta-teórica adquiere relevancia asentar con precisión la fotovoz en su marco de referencia que, como hemos argumentado, entendemos que es el paradigma crítico y ello, al menos, por dos razones. La primera porque los métodos y las técnicas de investigación siempre se basan en concepciones epistemológicas, siendo éstas las que les sirven de fundamento y orientación. El paradigma crítico está constituido por una ontología que es el realismo histórico, su epistemología es transaccional/subjetivista: hallazgos mediados por el valor y la metodología es dialógica/dialéctica. Además, hay que tener en cuenta, respecto a la "elicitación" visual fotográfica, que la foto-elicitación ya se había mencionado por primera vez en un artículo de Collier publicado en el año 1957 (Harper, 2002, p. 14) y que, en el desarrollo de ésta última han surgido nuevos enfoques, siendo uno de ellos, según la sistematización de Lapenta (2011), la fotovoz. A ello es 
necesario añadir que las decisiones a tomar al utilizar la foto-elicitación, como ha manifestado el último autor, giran en torno a quién va a seleccionar o a hacer las fotografías, a la elección de su contenido y a dónde se van a utilizar, y, desde nuestro punto de vista, ellas se hacen efectivas en la fase del proceso de una investigación en la que se recogen los datos, mediante técnicas, para dar respuesta al problema que ha originado la misma; por consiguiente, el enfoque de estos procedimientos específicos y el de la cuestión que se investiga están orientados por una concepción epistemológica, siendo la de la fotovoz la del paradigma crítico. Eso mismo se transfiere al ámbito pedagógico cuando la misma se aplica en el proceso de enseñanza y aprendizaje.

La segunda razón por la que consideramos importante la reflexión meta-teórica previa, que nos lleva a enmarcar la fotovoz en el paradigma crítico, es que el mismo aporta claridad a la confusión puesta de relieve en trabajos previos. Así, Doval (2015) ha señalado que a veces se confunde la fotovoz con la foto-elicitación, no siendo infrecuente que ambas aparezcan fusionadas, a lo que añade que "sigue existiendo una falta de consistencia en la identificación de la metodología en los estudios que aplican la fotovoz" (p. 246). Sitter (2017) ha manifestado que se dan una serie incoherencias al sostener que dicho enfoque de fotovoz es congruente con la investigación-acción participativa, específicamente en las áreas de posicionalidad del investigador, poder de decisión y tiempo dedicado al proceso de fotovoz (p. 41). Desde nuestra compresión, otra vez es el paradigma crítico el que orienta y rige estas áreas en la fotovoz, aportando coherencia, si bien éste no es el único enfoque en el que se puede realizar dicha investigación participativa. Martínez-Guzman, Prado-Meza, Tapia y Tapia (2018) han propuesto una relectura de este enfoque desde una perspectiva feminista post-estructuralista.

Al respecto, es el valor el que orienta cada corriente de pensamiento y el que introduce cierta diferenciación dentro de la misma concepción ontológica y epistemológica. La flexibilidad y adaptación a la que Wang y Burris (1997) aludieron estaría aquí presente.

Situada en el paradigma crítico, la fotovoz establece vinculación de técnicas de recogida de datos, de los métodos de observación y de encuesta, que incluyen las formas visual y verbal con diálogo desde el paradigma crítico. 


\subsection{El proceso metodológico de la fotovoz}

Una mayor concreción del proceso metodológico de la fotovoz se encuentra en un artículo posterior de Wang (2006) en el que se describe mediante una serie de nueve pasos, siendo intercambiables, en cuanto al orden, los dos primeros. Estos son:

1. Seleccionar y reclutar una audiencia destinataria de responsables políticos o líderes comunitarios.

2. Reclutar un grupo de participantes para la fotovoz. El tamaño ideal que se sugiere es entre 7 y 10 .

3. Introducir a los participantes en la metodología de la fotovoz y facilitar una discusión grupal acerca de las cámaras, el poder y la ética.

4. Obtener consentimiento informado.

5. Establecer el/los tema/s inicial/es para la toma de fotografías.

6. Distribuir las cámaras a los participantes y repasar cómo usarlas.

7. Proporcionar tiempo a los participantes para tomar las fotografías.

8. Reunirse para discutir las fotografías e identificar temas: se le puede pedir a cada participante que seleccione y hable sobre una o dos fotografías, las más significativas o las que más le gusten. Después los participantes pueden enmarcar las historias sobre las fotografías y tomar una postura crítica hacia ellas en términos de preguntas, a recordar mediante este acrónimo: SHOWeD. ¿Qué ves aquí? ¿Qué está pasando realmente? ¿Cómo se relaciona esto con nuestras vidas? ¿Por qué existe esta situación, preocupación o fuerza? ¿Qué podemos hacer al respecto? A continuación, los participantes codifican los temas, asuntos o teorías que surgen de sus fotografías. Estas tres etapas: selección de fotografías, contextualización o narración de historias y codificación de problemas, temas o teorías, se repiten en cada una de las rondas de fotografías tomadas por dichos participantes, siendo variable el número de rondas, dependiendo del proyecto o de otras consideraciones prácticas.

9. Planificar con los participantes un formato para compartir fotografías e historias con responsables políticos o líderes de la comunidad. (pp. 149-152)

Lo esencial en esta metodología de fotovoz es proporcionar cámaras a las personas para que puedan fotografiar sus realidades cotidianas. Las imágenes enseñan conceptos y pueden influir en la política. De ahí que las personas de la comunidad deban participar en la creación y definición de las imágenes que den forma a la política (Wang, 2006, p. 148). 


\subsection{Potencialidades y limitaciones de la fotovoz}

Los aspectos positivos que aporta la utilización del proceso de fotovoz se centran en la evaluación de necesidades participativas y de programas o prácticas sociales. Desde los trabajos precedentes, entre los que desatacamos el de Wang y Burris (1997), presentamos sus principales potencialidades:

- Posibilita la visibilidad de perspectivas experienciales del mundo de grupos o comunidades como vía para su transformación, siendo de destacar las de las poblaciones más vulnerables.

- Permite confrontar lo que los investigadores piensan que es relevante con otros puntos de vista que están presentes en la comunidad, asumiendo la legitimidad del conocimiento popular.

- Aporta a las personas una forma poderosa de representación de realidad vivida, como es la imagen visual y, entre ellas, a quienes no han adquirido aún la capacidad de leer y escribir, haciendo posible la comunicación.

- Incorpora diferentes escenarios comportamentales y sociales, así como de momentos y contenidos, de los que no hay conciencia a nivel colectivo.

- Puede mantener la participación comunitaria en el periodo entre la evaluación y la implementación de programas.

- Contribuye al conocimiento de las necesidades, recursos y bienes de las comunidades.

- Aporta beneficios tangibles e inmediatos a las personas y a sus redes sociales.

- Incluye en la investigación a participantes implicados en distintas prácticas, sin ser profesionales ni externos a las mismas.

- Detecta inadecuaciones en las teorías que fundamentan los programas o prácticas sociales desde la evidencia obtenida mediante el enfoque participativo.

- Evalúa las filosofías, enfoques y objetivos de los programas, reafirmándolos o redefiniéndolos,

- Estimula la acción social desde una mayor conciencia de los problemas.

La fotovoz, a la luz de las mismas fuentes, no sólo tiene ventajas sino también aspectos negativos. Las limitaciones más fundamentales son las siguientes:

- Ha de hacerse explícito el riesgo potencial para los participantes, que puede surgir por la intención política de la fotografía junto con el carácter crítico del diálogo para la acción.

- La dificultad de identificar las omisiones en la realización de las fotografías, dado que el juicio de las personas puede intervenir en muchos niveles de la representación. 
- Puede reproducirse una estratificación de clases más amplia por el control de los recursos.

- El análisis y la síntesis de los datos de las fotografías es difícil y complejo.

- Los ideales metodológicos pueden no coincidir con la realidad. (pp. 371-375)

Desde nuestro punto de vista, junto con los inconvenientes mencionados, la limitación esencial de la fotovoz es que la expresión del conocimiento obtenido desde ese diálogo y el consenso participativos no garantiza que pronuncie la realidad como es, con su incidencia en la efectividad de los programas o las prácticas que precisan todas las personas de la comunidad. Esta gran limitación tiene su raíz en la ontología y epistemología del paradigma crítico, ya especificadas, y podría superarse adoptando un marco más integrador, realmente participativo. Por ello, proponemos inscribir las técnicas y formas, visual y verbal, en el paradigma de investigación participativa articulado por Heron y Reason (1997). Su ontología subjetiva-objetiva es, realmente, participativa, su epistemología ampliada incluye cuatro formas de conocer: experiencial, presentativo, proposicional y práctico, en interrelación mediante la subjetividad y la intersubjetividad críticas, y su axiología es mejorar la realización personal y social, y esa de los ecosistemas de los que somos una parte.

\subsection{Presencia de la fotovoz en el ámbito de la educación}

En la investigación y la acción propia de diversos campos científicos se ha ido incorporando la fotovoz en las últimas décadas, estando entre ellos el de la educación. Su despliegue ha sido particularmente significativo, además de con los colectivos más vulnerables, con los niños, adolescentes y jóvenes. La revisión de Doval (2015) ha identificado las temáticas educativas en las que dicha metodología se ha aplicado, siendo éstas: "la educación en general, particularmente en estrategias de enseñanza y aprendizaje, discapacidad educativa e intelectual, autismo, la educación en orfanatos, el derecho a la educación, coeducación, educación inclusiva, educación universitaria, percepción de los espacios escolares y alfabetización" (p. 220).

En el contexto de la educación en Sudáfrica, Simmonds, Roux y Avest (2015) han mencionado que se ha utilizado esta metodología para provocar el cambio social y concienciar, junto a un uso específico en fenómenos como la niñez africana, las experiencias de pobreza de los maestros y los desafíos que enfrentan las maestras en los entornos educativos rurales (p. 35). 
No es usual el empleo de la fotovoz en España, siendo muy pocos los trabajos que, hasta la fecha, se han publicado. En el contexto internacional, Ciolan y Manasia (2017) han manifestado que la fotovoz sigue estando infrautilizada en el ámbito de la educación (p. 4).

Nuestra indagación, que pasamos a presentar, pondrá de manifiesto cuál ha sido la incidencia que ha tenido la fotovoz en una de las formas de la investigación cualitativa en educación.

\section{LA FOTOVOZ EN LA INVESTIGACIÓN EDUCATIVA DE ESTUDIO DE CASOS}

Desde la aplicación de la metodología ya explicitada pasamos a informar de los resultados obtenidos en las dimensiones del objeto de nuestra investigación.

\subsection{Incidencia, desarrollo temporal y canales de comunicación}

Aunque no es viable alcanzar la totalidad de la investigación educativa de estudio de casos mediados por fotovoz, sí consideramos que, dado el alcance de las bases de datos interrogadas y su cobertura, el resultado obtenido goza de amplia representación y significación con independencia de su cuantía. El número de documentos identificados ha ascendido a 40, de los que tan solo 3 son españoles. Estos datos ponen de manifiesto una muy escasa incidencia de este procedimiento en dicha investigación, evidenciándose una utilización incipiente en la educación española. Este resultado confirma la citada infrautilización de la fotovoz en el ámbito educativo (Ciolan \& Manasia, 2017), que entendemos que tiene que ver con el hecho de que la propia metodología es bastante reciente, lo cual va ser apoyado por el siguiente análisis. De ahí que nuestro trabajo pueda darla a conocer.

Si examinamos el desarrollo temporal de la fotovoz en esta investigación considerada, encontramos que el $90.0 \%$ de los documentos que la aplican ha sido publicados en la última década y un $60.0 \%$ en los últimos cinco años (2015-2019, básicamente, por realizarse la recuperación a principios del 2020).

Los canales de comunicación científica a través de los cuales se ha difundido son, fundamentalmente, las revistas, suponiendo los artículos un $77.5 \%$ del total de documentos. Las tesis doctorales también la han incorporado contribuyendo con un 15,0\%. Hay una presencia mínima del $2.5 \%$ de las tesis de maestría. Los trabajos presentados a congresos y 
conferencias internacionales aportan un 5.0\%. No ha faltado, por lo tanto, su presencia en los principales canales, formales e informales, de difusión de la ciencia.

\subsection{Orientación de la fotovoz en los estudios de casos, naturaleza de éstos y temáticas abordadas}

Aunque la fotovoz surge en el ámbito de la investigación, puede utilizarse, en cuanto procedimiento científico que es, con un propósito no sólo heurístico sino también didáctico. Al examinar el objetivo pretendido con la aplicación de la fotovoz en los estudios de casos en educación, encontramos que la intencionalidad investigativa ha estado presente en el $70.0 \%$ de los trabajos y la formativa en el $30 \%$ restante. No obstante, la primera conlleva capacitación de los co-investigadores. Apreciamos que ha habido un mayor desarrollo de la intención formativa si tenemos presente el resultado obtenido por Torre Y Murphy (2015) respecto a la utilización de foto-elicitación en educación, hallando que en un $10 \%$ de los estudios su propósito era pedagógico o evaluativo/de investigación-acción y en el $90 \%$ de investigación.

Conviene advertir que es frecuente un uso de la fotovoz combinado con otros procedimientos, sobre todo en su aplicación en la investigación, aunque también se ha dado en la didáctica.

El análisis de los tipos de casos presentes en las investigaciones educativas, ha puesto de manifiesto que un $57.5 \%$ de ellos eran proyectos o programas, en su mayoría en los niveles de educación formal, un $22.5 \%$ de los mismos, colectivos vulnerables o en riesgo de exclusión social, un $12.5 \%$ de aquellos, grupos de niños o alumnos de educación primaria y secundaria, y el $5.0 \%$ restante casos únicos.

La fotovoz se ha introducido de forma novedosa en los programas de enseñanza de varias disciplinas, entre las que indicamos las siguientes: inglés como segunda lengua, metodología de la investigación, ciencias medioambientales, ciencias de la alimentación y nutrición, química, didáctica de las ciencias, psicología comunitaria y trabajo social (Bowers, 2017; Boonekamp et al, 2019; Cook \& Quigley, 2013; Graciano, 2011; Lychyt, 2013; Matthews, 2013; a título de apoyo documental).

Desde la perspectiva heurística, algunas de las materias estudiadas en esos proyectos realizados han sido: educación para la salud, educación medioambiental, educación para el 
desarrollo sostenible, los huertos en el aprendizaje de los alumnos universitarios, creación de espacios pedagógicos, inclusión y roles de género (a título de muestra documental: Alaerts \& Wouters, 2019; Brand et al, 2019; Cole \& Altenburger, 2017; Derler, 2020; Perdersen et al., 2019; Yañez-Urbina, Figueroa, \& Soto, 2018).

Las temáticas atendidas en los estudios dirigidos a los colectivos vulnerables o en riesgo de exclusión social y a grupos de niños o estudiantes en niveles previos a la universidad, han sido, entre otras, las siguientes: prácticas escolares en la transición de la educación primaria a la secundaria, dificultades y barreras en la transición a la vida adulta, alfabetización visual en fotovoz, bienestar psicosocial, clima racial, experiencias espirituales, barreras en el aprendizaje de los estudiantes de educación superior, dificultades en el aprendizaje, visión de la escuela por los niños y participación de éstos en la escuela (Castro \& Manzanares, 2016; Doval, 2015; Graciano, 2004; HotchKins \& Dancy, 2017; Hoy, Parsons, \& Kovsholl, 2018).

Los estudios de tres casos únicos encontrados han abordado las experiencias de vida y el currículum de un joven-adulto con trastorno del espectro alcohólico fetal, la vivencia de la transición del hogar al jardín de infancia de una niña y la experiencia espiritual de un estudiante universitario de raza negra y gay (Brena et al., 2017; Lam, 2009; Means, 2014)

Estos resultados confirman gran parte de los temas educativos en los que la fotovoz ha sido aplicada (Doval, 2015), actualizándolos en esta forma de investigación cualitativa, así como el mayor uso de la fotovoz en la dimensión heurística respecto a la didáctica (Torre \& Murphy, 2015).

\subsection{Usos de los estudios de casos con fotovoz y aportaciones a la educación}

Al examinar la utilización dada a los estudios de casos de las investigaciones, desde la clasificación de referida en el apartado "metodología", comprobamos que todos ellos se han inscrito en el paradigma crítico y que la normatividad de sus valores ha tenido un carácter orientador en las investigaciones y las prácticas que se prescriben desde las mismas, impulsando el que sean atendidas. Por lo tanto, su uso es el desarrollo de la teoría normativa. Este hallazgo confirma la inscripción que hemos realizado, a nivel meta-teórico, de la fotovoz en el mencionado paradigma. Desde un nuevo análisis inclusivo de los trabajos en las categorías del mismo marco clasificatorio, hemos encontrado que el $42.5 \%$ de los 
mismos tienen un uso descriptivo y el $57.5 \%$ enfocado a la generación de hipótesis y al desarrollo de teorías.

Destacamos, seguidamente, algunas de las aportaciones que han surgido en la utilización descriptiva de los estudios de casos: visión de los niños de la escuela infantil y propuestas de mejora (Castro \& Manzanares, 2016); perspectiva de las estudiantes de educación secundaria sobre sus dificultades en el aprendizaje de las matemáticas (Harkness \& Stallworth, 2013); la voz de diversos componentes de una comunidad educativa acerca de los procesos y las practicas que contribuyen al éxito escolar en la implantación de una reforma educativa (Alaerts \& Wouters, 2019); descripción, desde la perspectiva de una profesora, de la aportación de la fotovoz al introducirla en un seminario de prácticas (Bowers, 2017); un discurso y una praxis sobre los temas de la vida cotidiana de niños y adolescentes en riesgo de exclusión social en el ámbito rural, con la valoración de la fotovoz para documentar la alteridad en la investigación y establecer la relación horizontal entre investigador e informantes (González, 2011); una descripción de las experiencias, habilidades y potencialidades de un joven-adulto con trastorno del espectro alcohólico fetal, con su inscripción en el curriculum (Brena, et al., 2017) y la perspectiva de estudiantes de raza negra sobre el clima racial existente en residencias universitarias (Hotchkins \& Dancy, 2017)

Aunque las investigaciones situadas en la categoría anterior no están completamente desprovistas de ideas y conceptos, el interés teórico es mayor en esas inscritas en la clase "generación de hipótesis y desarrollo de teorías". Entre las aportaciones de las mismas, expresamos las siguientes: comprensión profunda de los procesos de aprendizaje en la formación de profesores, de los factores que impulsan el desarrollo de competencias y de los obstáculos que se oponen al mismo (Brandt, et al., 2019); una ilustración de un enfoque alternativo fundado en las necesidades, desde una triple perspectiva, para comprender el autismo (Hoy, Parsons, \& Kovshoff, 2018); Un nuevo enfoque de aprendizaje basado en problemas para la alfabetización alimentaria sostenible de los estudiantes de educación secundaria (Derler et al., 2019); una compresión profunda de la espiritualidad de los estudiantes universitarios de raza negra y gais (Means, 2014); un mapa de causas y efectos de la baja e irregular participación, a corto plazo, en huertos llevados por estudiantes universitarios (Pedersen, Robinson, \& Surman, 2019) y la comprensión de la incidencia de la narración digital y la fotovoz en la motivación y la adquisición percibida del inglés en estudiantes de educación primaria y secundaria (Wright, 2015). 


\section{CONCLUSIONES}

La foto-voz es un procedimiento, específico y novedoso, que supone una vinculación de técnicas de recogida de datos, propias de los métodos de observación y de encuesta, basado y orientado por el paradigma crítico.

El desarrollo del mismo es bastante reciente, y escasa su incorporación a la investigación de estudio de casos en educación, desarrollándose, en su mayor parte, en los últimos 10 años. Su presencia en España todavía es mucho más incipiente.

La aplicación de dicho procedimiento en los estudios de casos en educación ha tenido una finalidad heurística y didáctica, con un amplio predominio de la primera, integrándose ambas en los trabajos de investigación-acción.

La fotovoz aporta un tipo de información a la que es muy difícil acceder desde otros procedimientos, tanto por su profundidad como por su riqueza, al incluir, también, experiencias, pasadas y presentes, sentimientos, ideas y creencias de las personas, mediante la fotografía y la suscitación del discurso, a nivel personal y colectivo, acerca de un asunto comunitario, con la pretensión de que esa perspectiva crítica y consensuada generada llegue a los responsables de los asuntos sociales y a los políticos para la propiciar la transformación de la comunidad. Este conocimiento experiencial constituye aportación propia y complementaria en la investigación cualitativa.

El conjunto de las investigaciones de estudios de casos en educación que han incorporado fotovoz ha aportado una comprensión crítica de distintas realidades educativas abordadas en las mismas, desde sus diversas perspectivas normativas, incorporando en ella los conocimientos experienciales.

Una parte importante de estas investigaciones educativas se han centrado en explorar la complejidad y singularidad de proyectos o programas, en gran parte situados en los distintos niveles de educación formal, tratando de obtener una comprensión en profundidad de los mismos y un conocimiento que informe el desarrollo de esas prácticas y de la política educativa. Otras investigaciones han aportado las perspectivas de colectivos vulnerables o en riesgo de exclusión social, posibilitando que sus voces sean escuchadas y difundidas. No han faltado en ellas las percepciones de los niños. Los casos únicos personales, aunque han estado presentes, ha sido muy escasos. El fomento de la fotovoz en estudios de casos 
que contribuyan a una educación inclusiva y al desarrollo sostenible constituye una línea de investigación valiosa.

La fotovoz, en el conjunto de estos estudios de casos, ha contribuido, en su uso en la investigación y la enseñanza, a la adquisición, por parte de sus participantes, de diversas competencias propias de la construcción y comunicación de conocimientos, acortando la distancia entre la teoría y la práctica.

Las propias limitaciones del paradigma crítico y, en particular de la investigación participativa que se realiza desde el mismo, inciden en la fotovoz. Sin entrar en ellas, por cuestión de espacio, apuntamos carencias en relación con las partes implicadas que se incorporan a la investigación o a la enseñanza y utilizan aquella; en la implicación de los participantes en todas las fases de la investigación, siendo el análisis de datos participativo una fase compleja y difícil de realizar; sesgos de representatividad, si no son casos únicos, en las características de quienes forman la muestra y por el tamaño; dificultades relacionadas con el control y el poder en la investigación, e impacto escaso o nulo de la misma, entre otras. Ellas han estado presentes en los estudios de casos de educación que han sido identificados.

Terminamos sugiriendo una incorporación de la vinculación de técnicas y formas, visuales y verbales, en el nuevo paradigma de la investigación participativa. Ella va a convertirse en una línea de nuestra futura investigación.

\section{REFERENCIAS}

Alaerts, L., \& Wouters, R. (2019). Photovoice as data collection method in a collaborative, visual, practiceoriented inquiry on junior high schools. European Congress of Qualitative Inquiry, Edinburgh. Retrieved from https://kuleuvencongres.be/enqi/articles/ecqi-full-papers.pdf.

Boonekamp, G. M., Dierx, J. A., Van Hove, P., \& Jansen, E. (2019). Interactive interviewing and imaging: Engaging dutch PVE-students in dialogue. Educational Action Research. DOI: 10.1080/09650792.2019.1693410.

Bowers, P. H. (2017). A case study of photovoice as a critical reflection strategy in a field seminar. Field Educator, 7, 1-10.

Brandt, J., Bürgener, L., Barth, M., \& Redman, A. (2019). Becoming a competent teacher in education for sustainable development: Learning outcomes and processes in teacher education. International Journal of Sustainability in Higher Education, 20(4), 630-653.

Brenna, B., Burles, M., Holtslander, L., \& Bocking, S. (2017). A school curriculum for fetal alcohol spectrum disorder: Advice from a young adult with FASD. International Journal of Inclusive Education, 21(2), 218229. doi:10.1080/13603116.2016.1193565. 
Castro A., \& Manzanares, N. M. (2016). Los más pequeños toman la palabra: La escuela infantil que a ellos les gustaría. Revista Complutense de Educación, 27(3), 923-941.

Cook, K., \& Quigley, C. (2013). Connecting to our community: utilizing photovoice as a pedagogical tool to connect college students to science. International Journal of Environmental \& Science Education, 8(2), 339357.

Ciolan, L., \& Manasia, L. (2017). Reframing photovoice to boost its potential for learning research. International Journal of Qualitative Methods, 16, 1-15.

Cole, L. B., \& Altenburger, E. (2017). Framing the teaching green building: Environmental education through multiple channels in the school environment. Environmental Education Research, 1-20.

Cordón, J. A., Alonso, J., Gómez, R., \& García, A. (2016). Las nuevas fuentes de información. Madrid: Pirámide.

Derler; H., Berner, S., Grach, D., Posch, A., \& Seebacher, U. (2020). Project-Based Learning in a Transinstitutional Research Setting: Case Study on the Development of Sustainable Food Products. Sustainability, 12, 233-248.

Doval, M. I. (2015). Una mirada crítica sobre la participación en la escuela a través de fotovoz. Estudio de un caso. (Tesis Doctoral inédita), Universidad de Vigo, Orense.

González P. (2011). "Tu mira la foto, pero no se la enseñes a nadie". Análisis de la práctica fotográfica, los discursos y las representaciones de niños y adolescentes en el contexto de talleres de fotografía participativa. Dos estudios de caso (Tesis Doctoral Inédita), Universitat Robira et Virgili, Tarragona.

Graziano, K. J. (2011). Working with English language learners: Preservice teachers and photovoice. International Journal of Multicultural Education, 13(1) doi:10.18251/ijme.v13i1.354

Harper, D. (2002). Talking about pictures: a case for photo elicitation. Visual Studies, 17(1), 13-16.

Harkness, S., \& Stallworth, J. (2013). Photovoice: understanding high school females' conceptions of mathematics and learning mathematics. Educational Studies in Mathematics, 84(3), 329-347.

Heron, J., \& Reason, P. (1997). A participatory inquiry paradigm. Qualitative Inquiry, 3 (3), 274-294.

Hotchkins, B. K., \& Dancy, T. E. (2017). A house is not a home: Black students' responses to racism in university residential halls. Journal of College and University Student Housing, 43(3), 42-53.

Hoy, K., Parsons, S., \& Kovshoff, H. (2018). Inclusive school practices supporting the primary to secondary transition for autistic children: Pupil, teacher, and parental perspectives. Advances in Autism, 4(4), 184196.

Lam, M. S. (2009) Crossing the cultural boundary from home to kindergarten in Hong Kong: a case study of a child's strategic actions. European Early Childhood Education Research Journal, 17(1), 125-145.

Lichty, L. F. (2013): Photovoice as a pedagogical tool in the community Psychology classroom. Journal of Prevention \& Intervention in the Community, 41(2), 89-96.

Lapenta, F. (2011). Some theoretical and methodological views on photo-elicitation. In E. Margolis \& L. Pauwels (Eds.), The Sage handbook of visual methods (pp. 201-213). Los Ángeles: Sage.

Margolis, E., \& Zunjarwad, R. (2018). Visual research. In N. K. Denzin \& Y.S. Lincoln (Eds.), The Sage handbook of qualitative research (pp. 600-622). Thousand Oaks, CA: Sage

Martínez-Guzmán, A., Prado-Meza, C. M., Tapia, C., \& Tapia, A. (2018). Una relectura de fotovoz como herramienta metodológica para la investigación social participativa desde una perspectiva feminista. EMPIRIA. Revista de Metodología de Ciencias Sociales, (41), 157-185.

Matthews, J. (2013). Enhancing food and nutrition curricula in higher education by assigning collaborative food 
system assessment projects. Journal of College Teaching \& Learning (TLC), 10(4), 245-254. doi:10.19030/tlc.v10i4.8121

Means, D. R. (2014). Demonized no more: The spiritual journeys and spaces of black gay male college students at predominantly white institutions. (Doctor of Philosophy Dissertation). Raleigh, North Carolina.

Pedersen, R. L., Robinson, Z. P., \& Surman, E. (2019). Understanding Transience and Participation in University Student-Led Food Gardens. Sustainability, 11, 1-26.

Schwandt, T. A., \& Gates, E. F. (2018). Case study methodology. In N. K. Denzin \& Y.S. Lincoln (Eds.), The Sage handbook of qualitative research (pp. 341-358). Thousand Oaks, CA: Sage.

Simmonds, S., Roux, C., \& Avest, I. T. (2015). Blurring the boundaries between photovoice and narrative inquiry: A narrative-photovoice methodology for gender-based research. International Journal of Qualitative Method, 14(3), 33-49.

Sitter, K. C. (2017). Taking a closer look at photovoice as a participatory action research method. Journal of Progressive Human Services, 28 (1), 36-48.

Torre, D., \& Murphy, J. (2015). A different lens: changing perspectives using photo-elicitation interviews. Education Policy Analysis Archives, 23 (111), 1-22.

Vázquez, G. (1981). N=1: Un nuevo paradigma de investigación pedagógica. Revista Española de Pedagogía, 39 (151), 3-13.

Wang, C. C., \& Burris, M. A. (1994). Empowerment through photo novella: portraits of participation. Health Education Q, 21(2), 171-186.

Wang, C. C., Ying, Y. Y., \& Ying, F. M. (1996). Photovoice as a tool for participatory evaluation: the community's view of process and impact. Journal of Contemporary Health, 4, 47-49.

Wang, C, C., \& Burris, M. A. (1997). Photovoice: concept, methodology and use for participatory needs assessment. Health Education and Behavior, 24 (3), 369-387.

Wang, C. C. (2006). Youth participation on photovoice as a strategy for community change. Journal of Community Practice, 14 (1-2), 147-161.

Wright, J. A. (2015). Through the looking glass: A case study of photovoice and digital storytelling with fourth grade English learners. (Degree of Doctor in Education Dissertation). Kennesaw State University, Kennesaw.

Yañez-Urbina, C., Figueroa, I., Soto, J., \& Sciolla, B. (2018). La voz en la mirada: Fotovoz como una metodología para explorar los procesos de inclusión-exclusión desde la perspectiva del estudiantado. Pensamiento Educativo, 55(2), 1-16. 